\title{
REVIEW
}

\section{Male sexual dysfunction in Asia}

\author{
Christopher CK Ho, Praveen Singam, Goh Eng Hong and Zulkifli Md Zainuddin
}

Sex has always been a taboo subject in Asian society. However, over the past few years, awareness in the field of men's sexual health has improved, and interest in sexual health research has recently increased. The epidemiology and prevalence of erectile dysfunction, hypogonadism and premature ejaculation in Asia are similar in the West. However, several issues are specific to Asian males, including culture and beliefs, awareness, compliance and the availability of traditional/complementary medicine. In Asia, sexual medicine is still in its infancy, and a concerted effort from the government, relevant societies, physicians and the media is required to propel sexual medicine to the forefront of health care.

Asian Journal of Andrology (2011) 13, 537-542; doi:10.1038/aja.2010.135; published online 6 June 2011

Keywords: Asia; erectile dysfunction; health; hypogonadism; male; premature ejaculation; sex

\section{INTRODUCTION}

Until recently, men's sexual health has taken a backseat in the planning and implementation of health care. When Viagra (sildanefil) burst onto the scene more than a decade ago, it opened up the floodgates for research and development on men's sexual health. As a result, a previously taboo subject was transformed into a popular topic, even in Asia. Being a conservative society, male sexual health was initially embraced with trepidation in Asia. In contrast, the more open culture of the West has seen significant progress in the field of sexual medicine. The purpose of this review is to examine the burden of men's sexual health in Asia and to identify issues that are unique to Asian men in order to develop strategies for the improvement of men's health care in Asia.

\section{EPIDEMIOLOGY}

Table 1 shows the prevalence of male sexual dysfunction in Asia.

Erectile dysfunction (ED)

ED is defined as the persistent inability to attain and maintain an erection that is sufficient to permit satisfactory sexual performance. ${ }^{1}$ The prevalence of ED in Asia varies between 9\% and 73\%. ${ }^{2-15}$ The wide variability in prevalence rates can be attributed to the method of assessment (i.e., self-reported or International Index of Erectile Function score) and the type of survey. For example, if subjects are recruited from a clinic, higher prevalence rates are attained than when subjects are recruited from the community. In the Asian Men's Attitudes and Life Events Study, the overall prevalence of ED was $6.4 \%$. $^{2}$ Alternatively, in Korea, when men greater than 20 years of age were recruited from primary care clinics and their International Index of Erectile Function score was determined, a prevalence rate of $32.2 \%$ was obtained. ${ }^{3}$ In Mainland China, the reported prevalence of ED is $38.3 \%{ }^{4}$ However, a prevalence rate of $9 \%-17.7 \%$ was obtained in Taiwan (China). ${ }^{5,6}$ In Thailand, the prevalence rate is $37.5 \%,{ }^{7}$ and in a population-based survey conducted in Singapore on men greater than 30 years old, a prevalence rate of $51.3 \%$ was obtained. ${ }^{8}$ In another study on the ageing population of Singapore, the prevalence rate was $73 \% .{ }^{9}$ The self-reported prevalence of ED in Malaysia is $26.8 \%$, but the prevalence rate obtained in another study was $69 \% .^{10,11}$

\section{Premature ejaculation}

Premature ejaculation is widely studied in the West; however, data from Asia are sparse. The International Society of Sexual Medicine defines premature ejaculation as a male sexual dysfunction that is characterized by ejaculation that always or nearly always occurs prior to or within about $1 \mathrm{~min}$ of vaginal penetration, the inability to delay ejaculation on all or nearly all vaginal penetrations and negative personal consequences such as distress, bother, frustration and/or the avoidance of sexual intimacy. ${ }^{16}$ The Global Study of Sexual Attitudes and Behaviors surveyed various aspects of sexual health among adults aged $40-80$ years old in 29 countries. In the aforementioned study, the overall prevalence rate was $30 \%$, and the highest rate $(30.4 \%)$ was obtained in Southeast Asia. Using these data, subgroup analysis was conducted on Asian men, and a prevalence rate of

Table 1 Prevalence of male sexual dysfunction in Asia

\begin{tabular}{lccc}
\hline & \multicolumn{3}{c}{ Prevalence (\%) } \\
\cline { 2 - 4 } Country & $\begin{array}{c}\text { Erectile } \\
\text { dysfunction }\end{array}$ & $\begin{array}{c}\text { Premature } \\
\text { ejaculation }\end{array}$ & Hypogonadism \\
\hline Malaysia & $26.8-69^{10,11}$ & $22.3^{18}$ & $18.5^{28}$ \\
Mainland China & $38.3^{4}$ & $19.5^{15}$ & - \\
Korea & $32.2^{3}$ & $27.5^{20}$ & - \\
Taiwan, China & $9-17.7^{5,6}$ & $13^{12}$ & $24.1^{58}$ \\
Thailand & $37.5^{7}$ & $23^{12}$ & - \\
Singapore & $51.3-73^{8,9}$ & $12^{12}$ & - \\
Hong Kong, China & $63.6^{57}$ & $29.7^{19}$ & $9.52^{59}$ \\
\hline
\end{tabular}


$20 \%-32.7 \%$ was obtained. ${ }^{14,17}$ In a study conducted in rural Mainland China, the prevalence rate was $19.5 \% .{ }^{15}$ However, in Malaysia and Hong Kong (China), the prevalence rate was $22.3 \%$ and $29.7 \%$, respectively. ${ }^{18,19}$ Moreover, in a recent study conducted in Korea, the self-reported prevalence rate was $27.5 \% .^{20}$

\section{Hypogonadism}

Hypogonadism is the clinical and biochemical entity previously known as andropause, Androgen Deficiency in the Aging Male and Partial Androgen Deficiency in the Aging Male. In 2005, a consensus was reached by the International Society of Andrology, International Society for the Study of the Aging Male and European Association of Urology, and the term 'late-onset hypogonadism' was defined as 'a clinical and biochemical syndrome associated with advancing age and characterised by sexual dysfunction and other typical symptoms and a deficiency in serum testosterone levels. This condition may result in significant detriment in the quality of life and adversely affect the function of multiple organ systems'. ${ }^{21}$ The International Society of Andrology, International Society for the Study of the Aging Male and European Association of Urology threshold for low total testosterone levels is $8 \mathrm{nmol} \mathrm{l}^{-1}$. When the total testosterone level is $8-12$ nmol $1^{-1}$, the free testosterone level must be less than $180 \mathrm{pmol} \mathrm{l}^{-1}$ to be considered low. ${ }^{22}$ Questionnaires are also available to aid in diagnosis. The Aging Male Symptoms score, Androgen Deficiency in the Aging Male score and a validated seven-question tool used by researchers in Hong Kong are useful screening tools. ${ }^{23}$ However, Androgen Deficiency in the Aging Male and Aging Male Symptoms questionnaires are of low specificity and do not correlate well with serum testosterone levels. ${ }^{24}$ The reported prevalence of hypogonadism in Asia (defined as total testosterone $<11 \mathrm{nmoll}^{-1}$ ) is $18.2 \%-19.1 \%{ }^{25}$ Data obtained from studies conducted in East Asia suggest that the prevalence of hypogonadism is between $7 \%$ and $47.7 \%$ in men aged 45-80 years old. ${ }^{23,26,27}$ In Malaysia, the prevalence rate in men over 40 years old was $18.5 \% .^{28}$

\section{PROBLEMS SPECIFIC TO ASIAN MALES}

In all nations, sound sexual health is a crucial issue for every man. Sadly, men in Asia are still suffering in silence. Despite its association with depression and diminished quality of life, sexual disorders are still largely ignored in Asia, the cradle of $60 \%$ of the world's population and the central focus of an economic boom. ${ }^{29,30}$ In contrast to the results described in the literature on the prevalence of sexual disorder in Asia, the actual numbers are significantly larger because the prevalence rate is based on self-reporting. In a study conducted in Taiwan (China), significant differences among prevalence rates were observed. Namely, compared to self-reporting, the prevalence rate was higher when it was based on the International Index of Erectile Function-5 score. In addition, among self-reported cases of ED, fewer than half of all men sought treatment. ${ }^{31}$ Asian men are also less likely to seek treatment than their Western counterparts. As a matter of fact, only $6 \%$ of men with ED in China sought effective treatment. ${ }^{30}$ Why does this phenomenon occur?

\section{Culture and beliefs}

Culture defines the role of men and women, how they relate to each other, their cultural group and the community. When addressing men's sexual health, several levels of culture and beliefs are involved, including the individual and his religion, community and nation. For males, masculinity is an important part of everyday life because it defines his role in society and among his peers. However, the concept of masculinity is undefined and varies among individuals and regions. Traditionally, masculine characteristics inhibit emotional expressiveness. Health-seeking behaviour among men implies a loss of status and control, and may damage their identity. ${ }^{32} \mathrm{Ng}$ et al. ${ }^{33}$ conducted a large multinational study, and demonstrated that Asian men associate masculinity with having a good job, being seen as a man of honour, being in control of their own life and being a family man. ${ }^{33}$ Having an active sex life, success with women and being physically attractive contributed less to the concept of masculinity. The low prevalence rate and non-medical seeking behaviour of Asian males with respect to men's health may be attributed to the aforementioned factors. Masculinity or maleness in sexual practice may be associated with sexual rights, sexual prowess and sexual performance. However, when their masculinity is threatened, men tend to keep quiet about it because they fear a loss of control and a loss of their cultural role more than poor health. ${ }^{34}$ As a result, Asian men tend to accept sexual disorder as part of ageing. Unfortunately, instead of seeking appropriate treatment, men seek alternative methods of self medication such as alcohol consumption to maintain their gender role. ${ }^{34}$

Does the negative attitude towards treatment among Asian men also exist among Asian immigrants in Western countries? In a previous study, the treatment seeking attitude of Western men and immigrant Asian men with cardiac pain was compared, and the results revealed that Asian men tended to experience greater anxiety and sought treatment markedly earlier. None of the immigrant Asian men considered seeking medical attention for chest pain as unmanly or a sign of weakness. In fact, some of these men regarded wisdom, education and responsibility for family and their own health as valued male attributes. Why does the treatment seeking attitude of indigenous and immigrant Asian men differ? Perhaps immigrants do not have the privilege of being sick for a long time because they do not have many relatives to depend on in a foreign country. Thus, these men must swallow their pride and risk being ridiculed as unmanly. ${ }^{35}$ Moreover, owing to the misconception that sexual disorder is not an important life-threatening matter, sexual dysfunction often goes untreated.

To further complicate matters, men in China regard ED as undignified. Discussing sexual disorders could result in embarrassment, especially if it involves oneself. Despite the fact that men with ED have a lower quality of life with respect to family life, work life, relationship with their sexual partner and general happiness, sexual dysfunction is a taboo and culturally restricted topic. ${ }^{36}$ Chinese men are not likely to discuss their sexual dysfunctions with anyone. However, if they do discuss their sexual health, they are more likely to talk to a personal friend or a traditional healer than a medical specialist. ${ }^{37}$ When Chinese men seek treatment from a conventional physician, it is more likely for comorbidities than sexual disorders. This is in contrast to Western men, who seek treatment for ED alone. ${ }^{30}$

\section{Compliance with health care}

Men view their bodies as machines and assume that they will function well. When faced with dysfunctions, men expect straightforward solutions. In general, long-term measures and lifestyle changes such as weight reduction, exercise, smoking cessation, healthy eating habits and a reduction in stress are not well received. Unlike women, who receive medical counselling throughout their reproductive life, men are less compliant with medical advice. This is an important aspect that must be considered when implementing health care policies for men's health. ${ }^{38}$ A study on Chinese female undergraduates in the USA revealed that females have a more positive attitude towards preventive 
care and have more regular checkups than males. ${ }^{39}$ In central Asia, $80 \%$ of all visits to primary health care providers are made by women and children, both in urban and rural settings. Men aged 50 years and older from urban areas of central Asia sought more medical consultations than rural men less than 50 years old. ${ }^{40}$ The observed differences in treatment seeking attitudes may be explained by differences in opportunity, current promotional campaigns that focus on the health of women and children, and general attitudes towards medical care. The fact that the life expectancy of men is 7 years shorter than that of women suggests that men's attitude towards health has taken a toll on their lives. $^{30}$

\section{Awareness}

Testosterone plays a pivotal role in men's health, and testosterone deficiency predictably produces the appearance of varied clinical scenarios involving different bodily systems, including the male reproductive system. ${ }^{41}$ Thus, for men with $\mathrm{ED}$, the attending physician has the opportunity to identify other associated illnesses before they become clinically florid. ${ }^{31}$ Among men with ED, 2\% face a major cardiovascular event within 12 months and $11 \%$ are affected within 5 years. ${ }^{42}$ Knowledge among Chinese men on androgen deficiency or partial androgen deficiency is insufficient. ${ }^{37}$ Unfortunately, knowledge of men's health is also lacking among physicians. Inadequate attention to this issue is one of the key reasons for the observed deficiency. ${ }^{30}$ Moreover, time constraints in the treatment of sexual disorders also contribute to the lack of knowledge. In particular, physicians must attend to other life-threatening illnesses, and the number of physicians is insufficient to meet patients' demands. ${ }^{32}$

Universal agreement on the clinical specialty responsible for men's health has not yet been obtained owing to the widely differing opinions on various aspects of men's health among a few specialty physicians. This is in sharp contrast to women's health, which is a specialty of gynaecologists. ${ }^{32}$ In a survey on physicians' attitudes towards men's health concepts in Asia, physicians generally associated men's health with diabetes and hypertension. Prostate diseases and ED were considered men's health issues less often. The majority of physicians believe that men's health is the domain of the urologist, and primary care physicians, endocrinologists and cardiologists are less important. The majority of physicians also agree that loss of normal function with age is inevitable, and the illness and conditions experienced by men as they age are predictable. Also, many physicians are fairly uncomfortable when dealing with male sexual problems owing to a lack of training and knowledge on sexual issues. ${ }^{32}$

\section{Alternative treatments/traditional medicine}

Asia is a melting pot of alternative and traditional medication. In China alone, traditional medication accounted for $30 \%-50 \%$ of the total consumption of medication. Moreover, in Malaysia, $65 \%$ of men felt that traditional medication was better than conventional medication. ${ }^{36}$

In China, a significant amount of scientific research has been conducted to evaluate the effectiveness of traditional herbs and acupuncture, and the effects on partial androgen deficiency. These studies suggest that positive outcomes can be obtained with alternative medicine and endorse the pivotal role of traditional methods in the treatment of androgen deficiency. Owing to the aforementioned results, many men seek traditional medicine to avoid embarrassing visits to medical specialists and the potential side effects of modern medicine. ${ }^{37}$

Many people turn to traditional medicine after becoming frustrated with the outcome of modern medicine. Patients are choosing to take more responsibility for their health by exploring a variety of self-regulatory and wellness practices. Alternative medicine encompasses these aspects, taking a holistic approach to patient's problems. Traditional practices such as acupuncture, acupressure, ayurvedic, yoga, herbal medicine, massage therapy, exercise, martial arts and spiritual healing are holistic approaches. These methods combine body, mind and spirit, and healings are achieved via the concept of energy rather than matter, as in modern medicine. Compared to the complexity of science, which is the basis of modern medicine, this concept is easily understood and comprehended, and is readily accepted because of its holistic approach.

The vast amount of ethnic groups, communities, cultures and daily traditional medicinal practices ensure that all aspects of men's health are included. Various traditional methods of penile augmentation have been developed, including the insertion of ball bearings under the penile skin or the insertion of semiprecious stones and gold bars through the glans. These practices are popular among working-class men in Southeast Asian countries because they are affordable and customers are promised that the wounds will heal in 4-5 days. In contrast, modern penile implants are more expensive and require hospitalisation. Approximately $1 \%$ of men applying for jobs in the shipping industry dabble in traditional methods of penile implants. ${ }^{43}$

Herbal medicine (phytotherapy) is the use of plants or plant extracts for therapeutic use. Phytotherapy is conducted to re-establish channels for the delivery of air, blood and nutrients, and to help balance the elements within the body. ${ }^{44}$ The main purpose of phytotherapy is to restore and realign the vital energy within the body, which is responsible for ageing, diseases and the deterioration of bodily functions. The healing theory adopts a holistic approach, in which an imbalance of energy in one system of the body affects other systems. Thus, herbal medicine is not specific to one system and is not meant to cure a single disease. In Western medicine, each pill is intended for a specific organ/ system; therefore, a patient may need a basket full of pills for multiple comorbidities. For this reason, traditional medicine is more attractive than conventional medication.

Various factors lead to the diminished use of conventional medication among Asian men. In particular, traditional medication is more congruent with Asian values, beliefs and philosophical orientation to health, as described above. ${ }^{45}$ Moreover, traditional medication is more affordable, as reported by the World Health Organization. Asia is a potpourri of various cultures and beliefs, and is blessed with an abundance of traditional medications. These medications can be obtained relatively easily because they are available in traditional medical establishments, markets and restaurants, and clinical consultations and prescriptions are not required. Inconvenience in the procurement of drugs is one of the most common reasons for the discontinued use of phosphodiesterase-5 inhibitor. ${ }^{31}$ Moreover, some modern therapies are difficult to administer. For example, alprostadil must be injected into the penis. Patient's fear towards the side effects of conventional medication is also an important factor, and many consumers believe that traditional medication is safer. ${ }^{45}$ Asian men also believe that their doctors are closed-minded, lack knowledge and do not understand or approve of alternative medication. In contrast, traditional practitioners are regarded as helpful, reassuring and positive. ${ }^{36}$

Most studies on traditional medication are conducted in vitro or in animals, and few studies are performed in humans. In addition, the active ingredients of these medications are not easily identifiable; thus, patients take a cocktail of mysterious chemicals of unknown efficiency and efficacy. Likewise, the effect of traditional medication can be variable because of changes in soil conditions and other environmental factors, which leads to inconsistent effects. 
As a result, optimal dosages are difficult to achieve. The flexibility or non-existence of quality control regulations in some parts of Asia further compounds this issue. ${ }^{36}$

\section{Access to health care}

Accessibility to health care in developing countries is a significant problem, and an inverse relationship between the distance to health facilities and the rate of utilisation has been observed. The aforementioned issue is a significant problem in Asia because the majority of nations are developing countries. Central Asian countries also face similar problems. In fact, some primary health care services cover catchment areas as large as $300 \mathrm{~km}$ between villages, and patients face extreme financial burden reaching health centres. Health care providers face similar difficulties, including prolonged travel time for home visits. ${ }^{40}$ Saroja et al. ${ }^{46}$ studied the Malaysian population, and noted that the utilisation of health care services was dependent on the presence of chronic illness, age and ethnicity. ${ }^{46}$ For instance, the Chinese utilize medical care less often because of their superior health status, under-reporting, reluctance, higher threshold of sickness and accessibility to traditional medicine. Poverty and educational status did not have an impact on health care-seeking behaviour.

Another problem that may affect men's health in the future is the affordability of modern medical treatment. The population in East Asia is ageing rapidly, and the economy may not be able to cope with an older population. In particular, between 2030 and 2050, the elderly population in major Asian countries is expected to increase to $15 \%$. If a country's economy cannot match its ageing population, sufficient resources may not be available for the elderly. ${ }^{47}$ Moreover, men's health campaigns will be significantly affected if end users are unable to afford blood tests, medications and other logistics pertaining to medical care.

The aforementioned results reflect the current health situation in Asia, and the term 'triple tragedy' accurately describes the circumstances (growing old before becoming rich, falling ill before getting old and escalating healthcare costs compound the tight development budget). ${ }^{48}$

Most prominent papers on men's health have been produced in the West, and studies conducted in Asia are relatively scarce. ${ }^{49}$ What factors contribute to this disparity? Asian men are physically, culturally and socially different from Western men; ${ }^{50,51}$ thus, results produced in the West may not be applicable to men in Asia. For this reason, statistics on Asian men must be obtained. Many different factors contribute to the scarcity of data on the sexual health of Asian men. For instance, the diverse culture present in Asia has been a double-edged sword. Namely, when studies are carried out in Asia, the results must be interpreted correctly and validated in many languages. Data analysis is an exhaustive affair that is compounded by disagreement among physicians as a result of misinterpretation caused by language barriers, culture, beliefs and economic and developmental status. Similarly, uniformity or standardisation in data collection is lacking; thus, data analysis is an uphill task. For example, different parameters have been used to measure serum testosterone levels. In two Asian studies, different cutoff values were used to evaluate the free testosterone concentration. In one study, a threshold of $0.023 \mathrm{nmol} \mathrm{l}^{-1}$ was applied, while in the other study, the cutoff value was set to $0.030 \mathrm{nmol}^{-1}$. To further complicate the situation, different assays were utilized, and the reliability and reproducibility of the assays varied. Owing to the differing biological effects of testosterone deficiency at different levels and ages, the results of the aforementioned studies were contradictory. ${ }^{41}$
Although previous studies confirmed the phytoandrogen effect, these investigations were conducted on animals, and good clinical trials on humans are scarce. ${ }^{41}$

\section{TREATMENT}

In addition to conventional modern medicine, Asians can utilize traditional and complementary medicine, which dates back to thousands of years. In terms of modern medicine, medications that are available for sexual dysfunctions are similar to those in the West. For ED, treatments include phosphodiesterase type 5 inhibitors, intracavernosal prostaglandin $\mathrm{E}_{1}$, medicated urethral system for erection, vacuum devices and penile prosthesis. The results of a previous study suggested that the safety and efficacy of sildenafil in men in Taiwan (China), was similar to that of the Western population. ${ }^{52}$ In a study on Asian diabetic patients treated with intracavernosal prostaglandin $E_{1}, 76.5 \%$ of ED patients reported satisfactory sexual activity. ${ }^{53}$ In another study conducted in Taiwan (China), the overall satisfaction with penile prosthesis surgery was $86.6 \% .{ }^{54}$ The armamentarium of traditional and complementary medicine available in Asia is shown in Supplement. However, strong studies with conclusive proof of the efficacy of these treatments have not been conducted.

For hypogonadism, testosterone replacement therapy can be given in the form of a gel, patch, oral or intramuscular injections. Testosterone undecanoate, a long-acting injectable testosterone, was recently introduced and has been studied in Korean patients with testosterone deficiency syndrome. The results indicated that testosterone undecanoate was effective, safe and tolerable. ${ }^{55}$ For premature ejaculation, psychological and behavioural therapy, local anaesthetic creams, SS creams, tramadol and selective serotonin reuptake inhibitors can be used. Dapoxetine, a new short-acting selective serotonin reuptake inhibitor that has fewer side effects than longer-acting selective serotonin reuptake inhibitors, has been recently introduced. In a study conducted on the Asia Pacific region, dapoxetine significantly prolonged intravaginal ejaculatory latency time and improved premature ejaculatory profile measures. In addition, the results suggested that dapoxetine was well tolerated. ${ }^{56}$

\section{FUTURE DIRECTIONS}

Compared to the West, data on male sexual dysfunction in Asia are relatively scarce, and sexual medicine has not been firmly established as a branch of medicine that warrants attention. However, sexual awareness has increased, and the Asia Pacific Society of Sexual Medicine has played a major role in the advancement of sexual health.

Asian males are clearly different from their Western counterparts with respect to biology, culture and beliefs. Currently, Asian patients are managed based on data obtained from the Western population. Asian researchers must develop decision aids and shared decision programs that are tailored to their local cultural context. Future research on men's sexual health should cover the psychobehavioural attitudes and mindsets of different ethnic groups in the Asian population. Profiling these males will assist in the development of personalized and effective healthcare plans that are based on shared decision models and evidence-based decision aids. ${ }^{48}$

Since time immemorial, Asians have been dabbling in traditional and complementary medicine. Owing to the diverse cultures present in Asia, many alternative treatments are available. However, these treatments have not been studied extensively. Most research on alternative medicine is conducted in vitro or is based on animal models. Therefore, the efficacy and safety of these treatments have not been proven. However, 
traditional and complementary medicine should be fully exploited, and more research should be conducted to sieve the good from the bad.

The mindset of Asians must be changed. Sex should not be viewed as vulgar, and physicians must directly confront sexual dysfunction, which is as important as diabetes and hypertension. The government also needs to allocate adequate funding for the treatment of sexual dysfunction. In many countries, medications such as PDF5 inhibitors are not subsidized by the government. Thus, most patients cannot afford treatment and suffer in silence. Societies such as the Asia Pacific Society of Sexual Medicine and Asia-Oceania Federation for Sexology should encourage the development of sexual medicine. Asian men should shed the taboos associated with sex and realize that sexual dysfunction is a disease that can be treated. To this end, the media can play an important role.

\section{CONCLUSIONS}

Male sexual health in Asia poses a challenge to healthcare providers because slightly different approaches must be adopted to address issues that are specific to Asians. Thus, significant progress on male sexual health must be made, and the vast resources of traditional and complementary medicine should be tapped to advance male sexual health in Asia.

1 Hatzimouratidis K, Amar E, Eardley I, Giuliano F, Hatzichristou D et al. Guidelines on male sexual dysfunction: erectile dysfunction and premature ejaculation. Eur Uro 2010; 57: 804-14.

2 Tan HM, Low WY, Ng CJ, Chen KK, Sugita M et al. Prevalence and correlates of erectile dysfunction (ED) and treatment seeking for ED in Asian men: the Asian Men's Attitudes to Life Events and Sexuality (MALES) Study. J Sex Med 2007; 4: 1582-92.

3 Cho BL, Kim YS, Choi YS, Hong MH, Seo HG et al. Prevalence and risk factors for erectile dysfunction in primary care: results of a Korean study. Int J Impot Res 2003; 15: 323-8.

4 Bai $Q$, Xu QQ, Jiang $\mathrm{H}$, Zhang WL, Wang XH et al. Prevalence and risk factors of erectile dysfunction in three cities of China: a community-based study. Asian J Androl 2004; 6: 343-8.

5 Chen KK, Chiang HS, Jiann BP, Lin JS, Liu WJ et al. Prevalence of erectile dysfunction and impacts on sexual activity and self-reported intercourse satisfaction in men older than 40 years in Taiwan. Int J Impot Res 2004; 16: 249-55.

6 Li MK, Garcia LA, Rosen R, Li MK, Garcia LA et al. Lower urinary tract symptoms and male sexual dysfunction in Asia: a survey of ageing men from five Asian countries. BJU Int 2005; 96: 1339-54.

7 Kongkanand A. Prevalence of erectile dysfunction in Thailand. Thai Erectile Dysfunction Epidemiological Study Group. Int J Androl 2000; 23 (Suppl 2): 77-80.

8 Tan JK, Hong CY, Png DJ, Liew LC, Wong ML et al. Erectile dysfunction in Singapore: prevalence and its associated factors-a population-based study. Singapore Med J 2003; 44: 20-6.

9 Chin CM, Khin LW, Quek P, Moorthy P, Lim P. Prevalence of erectile dysfunction in the ageing male population of Singapore: interim results of a nation-wide randomised survey. BJU Int 2002; 90 (Suppl 2): 38.

10 Khoo EM, Tan HM, Low WY. Erectile dysfunction and comorbidities in aging men: an urban cross-sectional study in Malaysia. J Sex Med 2008; 5: 2925-34.

11 Low WY, Khoo EM, Tan HM, Hew FL, Teoh SH. Depression, hormonal status and erectile dysfunction in the aging male: results from a community study in Malaysia. J Men's Health Gender 2006; 3: 263-70.

12 Feldman HA, Goldstein I, Hatzichristou DG, Krane RJ, McKinlay JB. Impotence and its medical and psychosocial correlates: results of the Massachusetts Male Aging Study. J Urol 1994; 151: 54-61.

13 Shirai M, Marui E, Hayashi K, Ishii N, Abe T. Prevalence and correlates of erectile dysfunction in Japan. Int J Clin Pract 1999; 102: 36.

14 Nicolosi A, Glasser DB, Kim SC, Marumo K, Laumann EO et al. Sexual behaviour and dysfunction and help-seeking patterns in adults aged 40-80 years in the urban population of Asian countries. BJU Int 2005; 95: 609-14.

15 Lau JT, Wang Q, Cheng Y, Yang X, Lau JT et al. Prevalence and risk factors of sexual dysfunction among younger married men in a rural area in China. Urology 2005; 66: 616-22.

16 McMahon CG, Althof SE, Waldinger MD, Porst H, Dean J et al. An evidence-based definition of lifelong premature ejaculation: report of the International Society for Sexual Medicine (ISSM) ad hoc committee for the definition of premature ejaculation. J Sex Med 2008; 5: 1590-606.
17 Moreira ED Jr, Kim SC, Glasser D, Gingell C. Sexual activity, prevalence of sexua problems and associated help-seeking patterns in men and women aged 40-80 years in Korea: data from the Global Study of Sexual Attitudes and Behaviours (GSSAB). J Sex Med 2006; 3: 201-11.

18 Quek KF, Sallam AA, Ng CH, Chua CB. Prevalence of sexual problems and its association with social, psychological and physical factors among men in a Malaysian population: a cross-sectional study. J Sex Med 2008; 5: 70-6.

19 Lau JT, Kim JH, Tsui HY. Prevalence of male and female sexual problems, perceptions related to sex and association with quality of life in a Chinese population: a population based study. Int J Impot Res 2005; 17: 494-505.

20 Park HJ, Park JK, Park K, Lee SW, Kim SW et al. Prevalence of premature ejaculation in young and middle-aged men in Korea: a multicenter internet-based survey from the Korean Andrological Society. Asian J Androl 2010; 12: 880-9.

21 Nieschlag E, Swerdloff R, Behre HM, Gooren LJ, Kaufman JM et al. Investigation, treatment and monitoring of late-onset hypogonadism in males: ISA, ISSAM, and EAU recommendations. Eur Urol 2005; 48: 1-5.

22 Lunenfeld B, Saad F, HoesI CE. ISA, ISSAM and EAU recommendations for the investigation, treatment and monitoring of late-onset hypogonadism in males: Scientific background and rationale. Aging Male 2005; 8: 59-74.

23 Wong SY, Chan DC, Hong A, Woo J. Prevalence and risk factors for androgen deficiency in middle-aged men in Hong Kong. Metabolism 2006; 55: 1488-94.

24 Beutel ME, Schneider H, Weidner W. Symptoms or complaints in the aging male which questionnaires are available? Urologe A 2004; 43: 1069-75.

25 Low WY, Khoo EM, Tan HM. Hypogonadal men and their quality of life. Aging Male 2007; 10: 77-87.

26 Li JY, Li XY, Li M, Zhang GK, Ma FL et al. Decline of serum levels of free testosterone in aging healthy Chinese men. Aging Male 2005; 8: 203-6.

27 Lin YC, Hwang TI, Chiang HS, Yang CR, Wu HC et al. Correlations of androgen deficiency with clinical symptoms in Taiwanese males. Int J Impot Res 2006; 18: 343-7.

28 Tan HM, Ng CJ, Low WY, Khoo EM, Yap PK et al. The Subang men's health research - a multiethnic community based study. Aging Male 2007; 10: 111.

29 Low WY, Khoo EM, Tan HM, Hew FL, Teoh SH. Depression, hormonal status and erectile dysfunction in the aging male: results from a community study in Malaysia. J Men's Health Gender 2006; 3: 263-70.

30 Sun Y, Liu Z. Men's health in China. J Men's Health Gender 2007; 4: 13-7.

31 Liao $\mathrm{CH}$, Chiang HS. Erectile dysfunction, testosterone deficiency, metabolic syndrome and prostatic disease in Taiwan. J Men's Health 2008; 5: 289-96.

32 Yates M, Low WY, Rosenberg D. Physician attitudes to the concept of "men's health" in Asia. J Men's Health 2008; 5: 48-55.

33 Ng CJ, Tan HM, Low WY. What do Asian men consider as important masculinity attributes? Findings from the Asian Men's Attitudes to Life Events and Sexuality (MALES) Study. J Men's Health 2008; 5: 350-5.

34 Bhui K, Chandran M, Sathyamoorthy G. Mental health assessment and south Asian men. Int Rev Psychiatry 2002; 14: 52-9.

35 Galdas P, Cheater F, Marshall P. What is the role of masculinity in white and South Asian men's decisions to seek medical help for cardiac chest pain? J Health Serv Res Policy 2007; 12: 223-9.

36 Low WY, Tan HM. Asian traditional medicine for erectile dysfunction. J Men's Health Gender 2007; 4: 245-50

37 Sun YH, Liu ZY. Men's health in China. J Men's Health Gender 2007; 4: 13-7.

38 Louis JG. Diagnostic approach to the aging male. World J Urol 2002; 20: 17-22.

39 Ray MS. Role of gender insurance status and culture in attitudes and health behavior in a US Chinese student population. Ethn Health 2001; 6: 197-209.

40 Cheryrl EC, Micheal B, Olga Z. The gender gap in primary health care resources utilization in Central Asia. Health Policy Plann 2002; 17: 264-72.

41 Lee BC, Tan HM. Late-onset hypogonadism: the Asian experience. J Men's Health 2008; 5: 297-302.

42 de Kretser DM. Determinants of male health: the interaction of biological and social factors. Asian J Androl 2010; 12: 291-7.

43 Terrence $\mathrm{HH}$. Putting men in the picture: problems of male reproductive health in Southeast Asia. In S22 Male Reproduction and Sexual Roles. Proceedings of the IUSSP XXIV Congress; 18-24 August 2001; Salvador, Brazil. IUSSP, Paris, France, 2001.

44 Yuen SH, Kwok FS, Raymond CC. Anti-aging herbal medicine-how and why can they be used in aging-associated neurodegerative diseases? Ageing Res Rev2010; 9: 35462 .

45 Wong LP, Tan HM, Low WY, Ng CJ. Traditional and complementary medicine (T/CM) in the treatment of erectile difficulties-experience from the Asian Men's attitudes to Life Events and Sexuality (MALES) study. J Men's Health 2008; 5: 356-65.

46 Saroja K, Kavitha S, Wah YL, Jemain AA, Tishya I et al. Factors contributing to utilization of health care services in Malaysia: a population based study. Asia Pac $J$ Public Health 2010; 21: 442-50.

47 Naohiro Y. Aging of the population in Japan and its implications to the other Asian countries. J Asian Econ 1997; 8: 245-61.

48 Tan HM, Horie S. Men's health in Asia. J Men's Health 2008; 5:265-6.

49 Low WY, Tong SF, Tan HM. Erectile dysfunction, premature ejaculation and hypogonadism and men's quality of life: an Asian perspective. J Men's Health 2008; 5: 282-8.

50 van Houten ME, Gooren LJ. Differences in reproductive endocrinology between Asian men and Caucasian men a literature review. Asian J Androl 2000; 2: 13-20.

51 Iwamoto T, Nozawa S, Yoshiike M. Semen quality of Asian men. Reprod Med Biol 2007; 6: 185-93. 
52 Chen KK, Hsieh JT, Huang ST, Jiaan DB, Lin JS et al. ASSESS-3: a randomised, double-blind, flexible-dose clinical trial of the efficacy and safety of oral sildenafil in the treatment of men with erectile dysfunction in Taiwan. Int J Impot Res 2001; 13: 221-9.

53 Tsai YS, Lin JS, Lin YM. Safety and efficacy of alprostadil sterile powder (S. Po., CAVERJECT) in diabetic patients with erectile dysfunction. Eur Urol 2000; 38: 177-83.

54 Chiang HS, Wu CC, Wen TC. 10 years of experience with penile prosthesis implantation in Taiwanese patients. J Urol 2000; 163: 476-80.

55 Moon DG, Park MG, Lee SW, Park K, Park JK et al. The efficacy and safety of testosterone undecanoate (Nebido $®$ ) in testosterone deficiency syndrome in Korean: a multicenter prospective study. J Sex Med 2010; 7: 2253-60.
56 McMahon C, Kim SW, Park NC, Chang CP, Rivas D et al. Treatment of premature ejaculation in the Asia-pacific region: results from a phase III double-blind, parallelgroup study of dapoxetine. J Sex Med 2010; 7: 256-68.

57 Siu SC, Lo SK, Wong KW, Ip KM, Wong YS. Prevalence of and risk factors for erectile dysfunction in Hong Kong diabetic patients. Diabet Med 2001; 18: $732-8$

58 Liu CC, Wu WJ, Lee YC, Wang CJ, Ke HL et al. The prevalence of and risk factors for androgen deficiency in aging Taiwanese men. J Sex Med 2009; 6: 936-46.

59 Wong SY, Chan DC, Hong A, Woo J. Prevalence of and risk factors for androgen deficiency in middle-aged men in Hong Kong. Metabolism 2006; 55: 1488-94.

\section{SUPPLEMENT}

Traditional medicines used in Asia

- $\quad$ Chinese yam (Dioscorea opposite)

- Eucomnia (Eucommia ulmoides)

- Ginseng (Panax ginseng)

- Fo-Ti-Tieng

- Dong-quai

- Deer Antlers (Cervi pantotrichum)

- Seahorse (Hippocampus kelloggii)

- Gingko (Gingko biloba)

- Tribulus terrestris

- Tongkat Ali (Eurycoma longifolia)

- Gambih

- Yohimbe (Pausinystalia yohimbe)

- Epimedium extract (Horny Goat Weed)

- Oat straw
- Oyster meat

- Nettle leaf

- Animal testicles

- Cayenne

- Astragalus

- Sarsparilla

- Licorice root

- Pumpkin seed

- Cuscuta

- Dendrobium

- Curculiginis

- Cornus cornus

- Cordyceps sinensis

- Cnidium seeds
- Cistanches

- Alpinia fruit

- Wild asparagus root

- Gynostemma

- Longan

- Lotus seed

- Lycium fruit (Fructus lycii)

- Morinda root

- Psoralea

- Rehmannia

- Schizandra fruit

- Walnut kernel 